\title{
[PREPRINT] Mind the Machines: Applying Implicit Measures of Mind Perception to Social Robotics
}

\author{
Zhenni $\mathrm{Li}^{*}$ (1) \\ Institute of Psychology and Ergonomics \\ Berlin Institute of Technology \\ Berlin, Germany \\ Joshua Pepe Woller* (i) \\ Institute of Psychology and Ergonomics \\ Berlin Institute of Technology \\ Berlin, Germany
}

\author{
Leonie Terfurth* \\ Institute of Psychology and Ergonomics \\ Berlin Institute of Technology \\ Berlin, Germany \\ Eva Wiese ${ }^{* \dagger}$ (1) \\ Institute of Psychology and Ergonomics \\ Berlin Institute of Technology \\ Berlin, Germany \\ eva.wiese@tu-berlin.de
}

\section{COPYRIGHT NoticE}

This is a preprint. It is not necessarily the final version of this article. This work has been submitted to the IEEE for possible publication. Copyright may be transferred without notice, after which this version may no longer be accessible.

\begin{abstract}
Beyond conscious beliefs and goals, automatic cognitive processes shape our social encounters, and interactions with complex machines like social robots are no exception. With this in mind, it is surprising that research in humanrobot interaction (HRI) almost exclusively uses explicit measures, such as subjective ratings and questionnaires, to assess human attitudes towards robots - seemingly ignoring the importance of implicit measures. This is particularly true for research focusing on the question whether or not humans are willing to attribute complex mental states (i.e., mind perception), such as agency (i.e., the capacity to plan and act) and experience (i.e., the capacity to sense and feel), to robotic agents. In the current study, we (i) created the mind perception implicit association test (MP-IAT) to examine subconscious attributions of mental capacities to agents of different degrees of human-likeness (here: human vs. humanoid robot), and (ii) compared the outcomes of the MP-IAT to explicit mind perception ratings of the same agents. Results indicate that (i) already at the subconscious level, robots are associated with lower levels of agency and experience compared to humans, and that (ii) implicit and explicit measures of mind perception are not significantly correlated. This suggests that mind perception (i) has an implicit component that can be measured using implicit tests like the IAT and (ii) might be difficult to modulate via design or experimental procedures due to its fast-acting, automatic nature.
\end{abstract}

\section{Index Terms}

social robotics; mind perception; agency; experience; implicit association; IAT; implicit measure; human factors; human-robot interaction; HRI

\footnotetext{
* Authors contributed equally to this research. ${ }^{\dagger}$ Corresponding author.
} 


\section{INTRODUCTION}

Sociality is central to human life, with social connections with fellow humans reducing stress and prolonging life expectancy [1]. In recent years social robotics, i.e., the use of robots designed to engage in meaningful social interaction with people [2], has become increasingly present in our private and professional lives. Consequently, our social interaction is not limited to human-human interaction anymore, but instead human-robot interaction (HRI) is on the rise.

To facilitate HRI cognitively and emotionally, research has focused on optimizing the design of social robots in terms of their appearances and functions [3]. Some researchers concentrate on examining behavioral differences between human-human and human-robot interactions, others focus on anthropomorphic features in robotics. Humanlike design is expected to enhance HRI by triggering social expectations and social principles of human-human interactions [2]. Though, high yet not perfect levels of anthropomorphism can also lead to unease and discomfort, particularly when they trigger categorical uncertainty or perceptual mismatch, making it challenging for the observer to decide whether they are interacting with a human or a robot [4], [5]. It is crucial to realize that engagement in social interactions depends not only on the other's factual social capability, but also on the human interaction partner's subjective belief whether the other agent "has a mind" (i.e. mind perception; [6]). When we perceive minds in others, we ascribe intentionality to them and are more likely to perceive them as individuals [7]. Mind perception also makes us feel socially more connected, fosters pro-social behaviors, and increases the social relevance of observed actions, which in turn leads, for instance, to better team performance [8]-[11]. Understanding mind perception in HRI is particularly important given its influence on social cognition [12]. Agents that fail to trigger mind perception, for instance, are treated as being incapable of experiencing complex emotions, are unlikely recipients of empathy, and make others feel less guilty when harming them [13].

\section{A. Theories of Mind Perception}

Gray et al. [6] suggest that mind perception can be best described by two dimensions - agency or the capacity to act and exert self-control and experience or the capacity to feel and sense. Agent assessments can vary independently along these two dimensions, with some of them yielding low agency and high experience ratings (e.g. babies) and others ranking high in both agency and experience (e.g., adults). Robots are traditionally ascribed low levels of experience and only low to medium levels of agency [6], [14]. Adding to the original theory, more recent theories propose that the perception of mind might be best described with at least three rather than two dimensions: Weisman et al. (2017), for instance, suggest to differentiate between physical, cognitive and emotional aspects of mental life by sorting capacities according to their relations to the body, the mind or the heart [15]. Malle (2019) proposes that mind perception is best described through the dimensions affect (unintentional emotional capacities), moral 
and social cognition (capacities connected to the simulation of ones own and others' mind) and reality interaction (capacities connected to action and communication) [16].

Regardless of the exact number and nature of the dimensions involved in mind perception, researchers agree that different features of a perceived agent (i.e., triggers) make it more or less likely that mind is attributed to an agent [11]. This means that mind cannot only be ascribed to agents that actually "have a mind" (e.g. humans) but also to agents without an actual mind (e.g., robotics agents) or with ambiguous mind status (e.g., dogs) [6]. It also implies that mind perception can be systematically triggered by manipulating either an agent's physical appearance, its behavior or the observer's belief about the agent's mind status (via instruction).

Using subjective measures (e.g., questionnaires) and abstract stimuli, mechanisms of mind perception have been examined via manipulation of physical or behavioral features of nonhuman (i.e., anthropomorphism) or human agents (i.e., dehumanization) [17]-[21]: nonhuman agents resembling humans by displaying prototypical human facial features and proportions trigger mind perception and activate brain areas involved in social cognition within milliseconds [22]. When physical appearance or behavior is not prototypical, however, it induces categorical uncertainty (e.g., uncertainty whether an agent is human or nonhuman) associated with negative emotional reactions (i.e., Uncanny valley effect) and reduced cognitive performance across different tasks [5], [20], [23]-[26]. Previous studies have shown that human behavioral characteristics like predictability, goal directness and display of emotional reactions induce mind perception even when being displayed on abstract stimuli like geometric shapes [27] or robotic agents [28], [29]. The human brain is so attuned to behavioral signs of human likeness that even low-level motion kinematics, such as acceleration/deceleration or timing patterns during pointing reliably lead to attribution of mind [30]. Although these studies uniformly highlight the importance of mind perception for social interactions, HRI research has either neglected this concept when evaluating human-robot interactions or has exclusively focused on the use of subjective measurements as basis for the evaluation. The following section discusses potential issues that are associated with the exclusive use of explicit measures to examine mind perception and proposes an alternative that allows researchers to measure mind perception more implicitly.

\section{B. Measuring Mind Perception}

a) Bias in Explicit Measures: Mind perception can be measured explicitly (directly addressing conscious representations) and implicitly (indirectly addressing automated processes) [31]. So far, most of the empirical works on mind perception towards robotic agents has been focused on explicit measures assessing conscious attitudes based on rating scales and self-report [6], [15], [16], [32], [33]. While such direct methods provide researchers with seemingly straight-forward access to people's conscious attitudes, they can be biased in multiple ways. Macdorman et al.(2009) argued that in some cases participants might not be aware of the attitudes that ultimately affect their 
behavior [34]. If a person is consequently unsure of their attitudes or the reasons behind them, they tend to fall back on known popular explanations and evaluations and thus would not report their actual personal attitudes. Another source of bias in explicit measures is associated with social desirability. It may occur when participants face real or perceived social pressure, conceal their real feelings, and choose to align their attitudes with the perceived consensus. Furthermore, explicit measures require conscious processing and as such can be biased by pre-existing knowledge or stereotypes (e.g., about human and nonhuman agents in general). Consequently, the risk is high particularly in short one-time experiments - that the variation in the data is mainly due to peoples' pre-existing conceptions, rather than due to experimental manipulation or the to-be-examined robot features.

b) Dual Process Models: To overcome the biases associated with explicit measures, researchers consider using implicit measures to grasp humans' underlying automatic evaluation of an object. Benefiting from minimized potential biases and good internal reliability, measures such as the implicit association test (IAT) [35] and the Go/NoGo Association Task (GNAT) [36] are applied in various settings. It is worth noting that perception and behaviour in general are affected by implicit attitudes and explicit reasoning processes in tandem [37]. Therefore, mind perception towards non-human agents can be best described by dual-process models [38], providing a unified account of both implicit and explicit evaluations of the same object. Dual-process models propose that fast, automatic and implicit cognitive evaluations can be triggered by low-level features of a stimulus (e.g., a robot with a human-like body or a robot that behaves as if it were in pain), which in turn can be further influenced (but are not necessarily bound to) by slower, explicit, resource-demanding, conscious reasoning processes (e.g., a verbalized thought that a robot is a mere machine, and thus incapable of feeling pain). This suggests that the overall attitude a person endorses not only depends on the cognitive capacity to form and retrieve an explicit attitude but also whether the implicit attitude overrides the explicit attitude. They are both connected, but qualitatively separate [39]. Especially when certain evaluations can be judged as reprehensible and thus are susceptible to the influence of social desirability, implicit measures often provide a more accurate explanation of human behaviour [35].

c) Implicit Measures in Social Robotics: While explicit measures have been the most frequent method used in the research area of social robotics, some scientists have acknowledged the importance of implicit measures. A few studies have been using reaction time-based measures, either addressing more general questions of implicit attitudes towards automation technology [40] or directly focusing on robotics agents [34], [41]-[43]. Using the original IAT stimuli, photos [43] or silhouettes [41], [42] of robots vs. human agents for object stimuli, a stronger implicit negative association with robotic agents compared to human agents was shown. Additionally, humans seem to have more implicit pleasant associations with other humans compared to robots and implicitly associate robots with threats [34].

A study by Spatola et al. compares the semantic distance between the concepts of humans and robots towards 
words describing secondary and primary emotions in a reaction time based paradigm [44]. Their results indicate that implicit attitudes predict explicit attitudes towards robots and suggest the implicit measure to be better predictors of a future behaviour towards the robot than explicit measures. Moreover, an implicit measure finding different emotions towards robots with different degrees of anthropomorphism has been introduced [45]. Złotowski and colleagues propose a dual process theory perspective on anthropomorphism after comparing the effects of humanlike characteristics ascribed to a robot in explicit and implicit measures [46]. The application of 5 mostly rating based tests of implicit ascription of mind (e.g., Sally-Anne-Test) towards robotic agents suggests that implicit and explicit mind ascription are not associated [47].

These studies suggest that implicit behavioral measures comparing attitudes towards human and robotic agents in general are promising and can lead to meaningful results. Implicit measures of mind perception specifically, however, have not been sufficiently reported in the literature yet. On that ground, more research using implicit measures to detect human's automatic evaluation of social robotics is needed.

\section{AIM OF STUDY}

Due to the current lack of implicit measures in mind perception studies, we are aiming to extend the available range of tools by introducing an additional easy-to-use and easy-to-access measure to examine mind attribution towards robotic agents. Specifically, we describe the development of an IAT measuring mind perception in social robotics (MP-IAT) based on the two-dimensional approach to mind perception, differentiating between agency and experience [6]. Thereby, we expect to contribute to a more complete understanding of mind perception in HRI. By applying the MP-IAT to already existed robot stimuli, we are seeking to gain more insights into the implicit perspective of mind attribution towards robotic agents. Additionally, we examine the relation between explicit and implicit mind attribution by correlating the results of the MP-IAT with the outcome of explicit mind perception ratings towards the same agents.

In line with previous reports using explicit measures to examine mind perception in HRI [6] we expect the implicit attribution of agency and experience (using the MP-IAT) to be higher towards human than robot agents. Regarding the size of those effects, we hypothesize a stronger effect for the experience dimension than the agency dimension. For the explicit measures, we assume that robots will on average be rated to possess low agency and low subjective experience. Humans, on the contrary are expected to be rated very high along both dimensions. With reference to mixed results for the correlation of implicit and explicit measures [48], we do not proclaim any specific relations between implicit and explicit perception of mind. To gain further insights their correlation will be explored. 


\section{Methods And Materials}

\section{A. Participants}

53 participants in total completed this study. Before data analysis, the results from 2 participants were eliminated after failing attention checks (individual IAT error rate two standard deviation above sample mean). Therefore data from a total of 51 eligible participants was used in the further analysis (29 women, 21 men, and the overall $\mathrm{M}_{\text {Age }}$ $=47.20$ years old with an age range from 21 to 74 years). More than half of the participants had no or only limited previous experience with robots $(45.1 \%$ none, $11.8 \%$ on a single occasion, $35.3 \%$ on some occasions, $5.88 \%$ on frequent occasions). $96.1 \%$ stated to be fluent in English language, the rest did not answer the question. Participants were recruited via Amazon Mechanical Turk (MTurk), an online crowdsourcing marketplace. A location restriction on MTurk has been placed so that only participants who are located in the United States, Canada and the United Kingdom were eligible for this study. This was considered to ensure that all study participants came from English speaking countries and would also share similar western cultural values. In addition, only MTurkers with an approval rate of at least $85 \%$ (percentage that their participation's have been approved by past requesters) were allowed to participate. Successful completion of the experiment was compensated with a $\$ 2.00$ cash reward through the platform.

\section{B. Implicit Association Test Method}

The implicit association test (IAT) by Greenwald and colleagues [35] is a widely used reaction time based method to measure participants' underlying implicit attitudes through the strength of association between different targets (e.g., Black people vs. White people, women vs. Men) and attributes (e.g. good vs. bad, natural sciences vs. social sciences). The rationale is that participants would be able to categorize stimuli which are in line with one's associations (congruent trials) faster than when presented stimuli and associations are less expected (incongruent trials).

In most implementations of the IAT, including our study, participants complete 7 blocks of categorization tasks. In practice blocks (blocks 1, 2, and 5), single stimuli (words or pictures) are categorized by a key press (e.g., words that belong to the concept "good" = A, words that belong to the concept "bad" = L). In the combined blocks (blocks 3, 4, 6, and 7), concept and attribute stimuli are presented consecutively in random order. In congruent trials, categorization keys are assigned so that stimuli with strong expected association are mapped to the same key (i.e., stimuli that belong to the concept of flowers + "good" = A, stimuli that belong to the concept of spiders + "bad" = L), whereas in the incongruent condition keys are assigned to the combination of stimuli with lower expected association. 
Upon completion, an effect size measure (D-score) is calculated by subtracting the average time it takes to categorize stimuli in the congruent trials and the average time it takes to categorize stimuli in the incongruent trials, divided by the pooled standard deviation (for congruent and incongruent conditions). Zero in D-score indicates no bias between target and stimuli. A negative D-score implies an implicit association or attitude in the direction of expectation (e.g. spiders are implicitly stronger associated with "bad"; flowers are implicitly stronger associated with "good"). Cut-off values of D-scores of 0.15 ("slight association"), 0.35 (“moderate association"), and 0.64 ("strong association") are widely used [49].

IAT scores have been shown to be on average only moderately correlated with corresponding explicit measures, supporting the perspective that related, but separate constructs are assessed by those methods [48].

\section{Tasks and Procedure}

The experiment was programmed in Python 3 and JavaScript using the PsychoPy environment. The online study was hosted on pavlovia.org. After starting the experiment, the participants were presented with demographic questions and basic instructions and granted their informed consent. Upon completing the demographics, participants advanced to the two-part MP-IAT.

\section{1) Implicit Association Test:}

a) Attribute Stimuli: The selection of attribute stimuli for the MP-IAT, i.e., words belonging to either facet of mind perception, was based on previous research. Agency word selection was inspired by the Agency IAT conducted by Campbell et al. in a study about narcissists' self-perception [50]. Major adaption was necessary due to a different underlying definition of agency, as the original selection of words combines agency and the personality trait of extraversion. Overall, nine words that were considered to primarily describe agency in social behavior (e.g., "assertive", "dominant", "submissive") were dropped, and new agency-related words as well as their respective antonyms were introduced instead (e.g., "goal-oriented" vs. "remote-controlled" and "thoughtful" vs. "mindless"). These better represent agency as the capacity to independently initiate and control one's actions. Table I lists the attribute stimuli for the experience dimension, based on Smeding's verbal stimuli for rationality and emotionality, originally used in an IAT exploring gender stereotypes [51]. For our purposes, rationality, i.e. the capacity to reason with pure logic, was considered to represent low subjective experience, while emotionality, i.e. the capacity to perceive and process subjective feelings, was considered to represent high subjective experience.

b) Target Stimuli: While attribute stimuli were verbal in nature, the agent targets were represented by different photographs of humans and robots (See Figure 1 for an example of the agents). The "human" category was represented by images of six adults of different genders and races, while robots were represented by images of six 
TABLE I

ATTRIBUTE STIMULI USED IN THE MP-IAT.

\begin{tabular}{llll}
\hline $\begin{array}{l}\text { Low } \\
\text { agency }\end{array}$ & $\begin{array}{l}\text { High } \\
\text { agency }\end{array}$ & $\begin{array}{l}\text { Low } \\
\text { experience / } \\
\text { Rationality }\end{array}$ & $\begin{array}{l}\text { High } \\
\text { experience / } \\
\text { Emotionality }\end{array}$ \\
\hline passive & active & consistent & empathic \\
mindless & thoughtful & deductive & perceptive \\
inconsiderate & ethical & logical & intuitive \\
programmed & goal-oriented & objective & sensitive \\
inhibited & outspoken & reasonable & subjective \\
remote-controlled & planful & rational & emotional \\
\hline
\end{tabular}
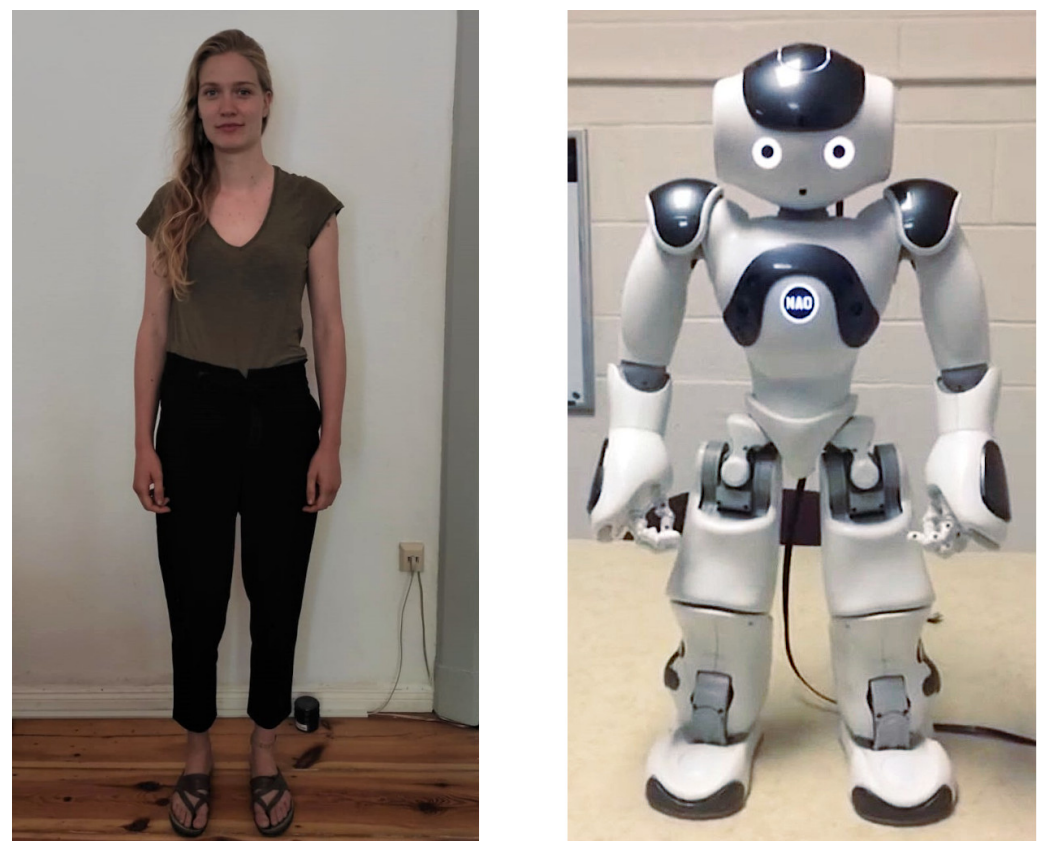

Fig. 1. Sample target stimuli showing a human and a robot (NAO). Consent for publishing both images was granted.

humanoid robots ${ }^{1}$. Only robots possessing sufficient visual similarity to the included reference specimen (NAO, Fig. 1 ) were chosen. For each robot, visual similarity was inferred via the image collection of ABOT-Database [52]. For this study, we required robots to possess four limbs and at least eyes as facial features. Robots and humans were presented in full-body pictures in an upright standing position directly facing the camera. To maximize the similarity of stimuli, robots were placed on a digitally added "floor" to stand on. Images of both agent types were taken against a neutral background.

c) Procedure: Information about the experimental procedure of the IAT and examples for the categorization of stimuli were viewed in a self-paced manner. To help participants get familiar with the selection of stimuli, an overview of the robots and humans that later would appear in the study was given. This lasted at most $45 \mathrm{~s}$ or until

\footnotetext{
${ }^{1}$ Robotic agents chosen: RoBoHon (Sharp Electronics), ALPHA 1P (UBTECH Robotics), iCUB (RobotCub project), Roboy (Devanthro $\mathrm{GmbH}$ ), NAO (SoftBank Robotics), KIBO (Korea Institute of Science and Technology)
} 

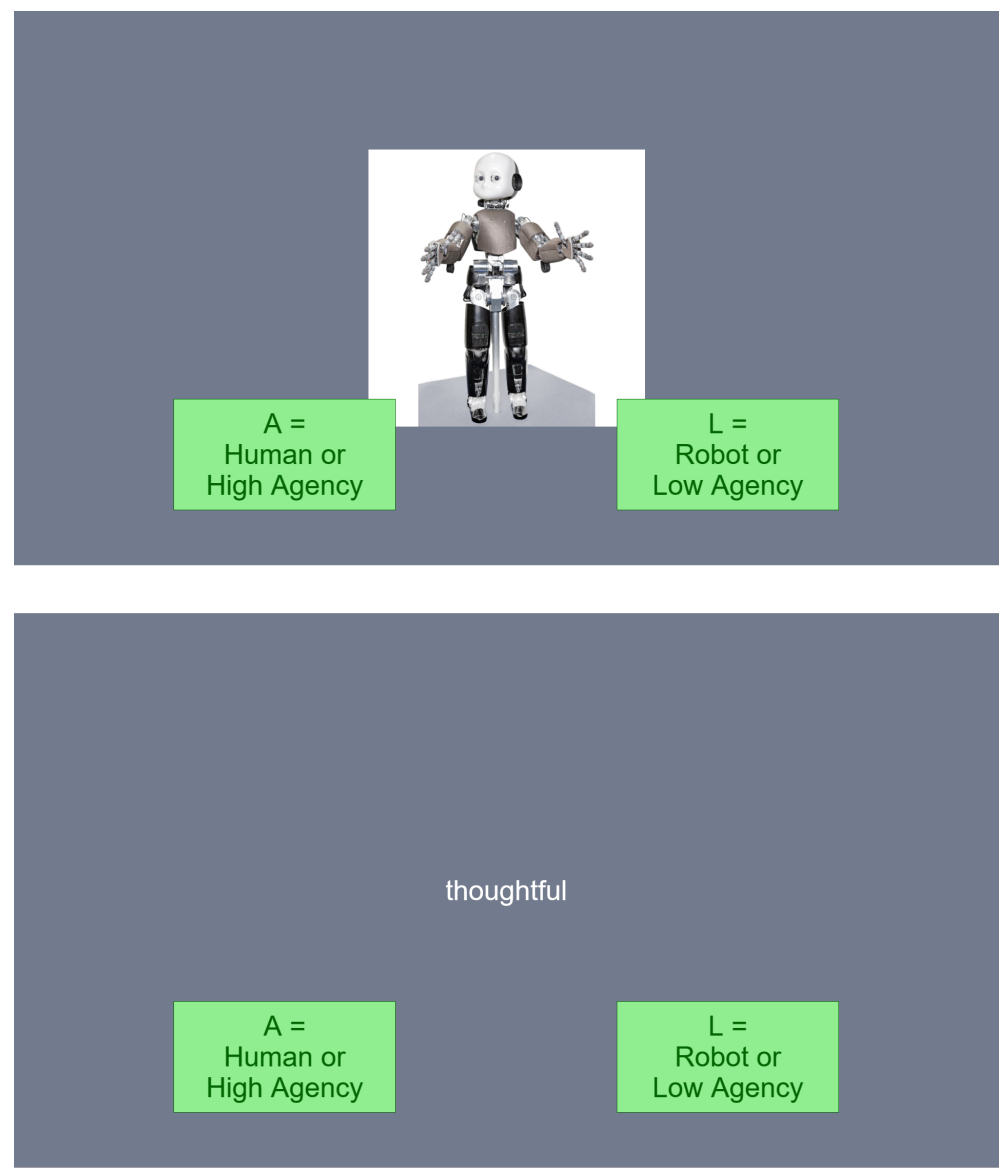

Fig. 2. Sample of screen display in congruent combined IAT blocks. Full display of IAT sequence available in video (S1).

a key response was given. All stimuli in the study were presented on a medium gray background (see Fig. 2 for an example). Text was displayed in white Arial font. Font size was automatically adjusted depending on the user's screen size. Participants were presented with two IATs that differed in their attributes, but not in their target stimuli (Human/Robot vs low/high Agency and Human/Robot vs low/high Experience). The order in which participants were presented the IATs was randomized. Within each IAT, the order of congruent and incongruent blocks was randomized as well, leading to 8 different block orders in total. Each IAT consists of 7 blocks, with the practice blocks 1, 2, and 5 consisting of 20 trials each. Practice blocks introduced relevant stimuli and categories as well as the assignment of response keys to them. Blocks 3, 6 with a length of 20 trials and 4, 7 with a length of 40 trials were combined blocks. Before each block of trials, the assignment of categories to response keys was presented on a separate slide. In the lower left corner, categories (i.e., 'Robot or Emotionality') associated with the 'A' key were shown, while those belonging to the 'L' key (e.g. 'Human or Rationality') were displayed in the lower right corner, each in a green text box. Participants were asked to use their left and right index fingers to press the ' $A$ ' and 'L' keys.

At the beginning of each trial, a fixation cross was presented for $150 \mathrm{~ms}$. Then, the stimulus appeared and 
TABLE II

Mind Perception Questionnaire For DifFEREnt Agents (Human \& Robot).

\begin{tabular}{ll}
\hline Agency & Experience \\
\hline Agent can: & Agent can: \\
...have thoughts. & ..feel love. \\
...tell right from wrong. & ...feel tired. \\
...understand others' minds. & ...feel pleasure. \\
...plan future actions. & ...feel happy. \\
...set goals. & ...feel pain.. \\
...exercise self-control. & ..ffeel stress. \\
...uphold moral values. & \\
\hline
\end{tabular}

participants categorized the stimulus using the assigned keys. The key category assignment remained visible during the entire duration of the trial. Stimuli remained on the screen until a response was provided. Only during practice blocks, each trial was followed by feedback if the classification was incorrect (i.e., the word 'false' was presented in red at the screen centre for 1s). Participants were provided with information about their average classification accuracy and response time at the end of each block, and the feedback remained on screen until the next block was started with a key press. After the first IAT, a mandatory break of at least two minutes was inserted. Participants could then resume the experiment through the press of a button. The second IAT was identical in structure to the first one, but featured the category (agency or experience) not assigned during the first test. Following both IATs, participants were asked to fill in a short questionnaire assessing explicit mind perception towards human and robots.

\section{2) Mind Perception Questionnaire:}

a) Items: Based on research from Malle [16] and Weisman et al. [15] we developed a short scale to measure the explicit attribution of mind perception, encompassing 15 items in total (see Table II)). To ensure comparability with our implicit measures we picked items clearly assignable to the agency and experience dimensions as defined by Gray and Gray [6]. Experience was assessed by asking the subject about the assumed capability of an agent to feel specific affective states. Agency was represented by items referring to the capability to perform independent actions as well as an agent's moral ability. Agreement with 7 items related to agency (e.g. "A human can set goals") and 6 items related to experience (e.g. "A robot can feel love") was evaluated by each participant on an 8-point Likert scale from 1 (not at all) to 8 (totally).

b) Procedure: Only a single statement at a time was shown on the screen. Participants indicated their agreement on a visual 8-point numeric rating scale. After hitting the confirmation button for the rating, they were advanced to the next statement. Ratings could not be changed at a later point. Aggregate scores for each combination of agent type and facets of mind perception (e.g., experience in robots) were calculated by the average score of the corresponding items. Upon completing the questionnaire, participants were thanked for their participation and 
received further information about the robots shown in the study. They were also provided with a code to collect their payment on Amazon MTurk.

\section{Analysis}

We used R 4.1.2 to pre-process and analyse the data. The IAT package was used to calculate D-scores, applying the scoring algorithm recommended by Greenwald, Nosek, and Banaji [53]. Error latencies were replaced by the block mean $+600 \mathrm{~ms}$, extreme values (latencies smaller than $400 \mathrm{~ms}$ ) were deleted and block SD were calculated including error trials. In line with the content of this study a negative D-score indicates an association between robots and low mind perception and between humans and high mind perception, whereas a positive D-score indicates the opposite bias (robot and high mind perception; human and low mind perception). Plots were created in Python 3 , using the Seaborn and matplotlib libraries, D-Scores were visualised using raincloud plots [54]. 


\section{RESULTS}

\section{A. Implicit Mind Perception}

One-sample t-tests were conducted to assess differences in implicit attribution of agency and experience towards human and robotic agents. Both implicit agency D-score $(\mathrm{M}=-0.40, \mathrm{SD}=0.31, \mathrm{t}(50)=-9.37, p<0.001)$ and implicit experience $\mathrm{D}$-score $(\mathrm{M}=-0.15, \mathrm{SD}=0.36, \mathrm{t}(50)=-2.82, p=0.006)$ differ significantly from zero (see Fig. 3). A paired-sample t-test revealed a significant difference between results for agency and experience ( $M=$ $-0.26, \mathrm{SD}=0.48, \mathrm{t}(50)=-3.82, p<0.001)$.

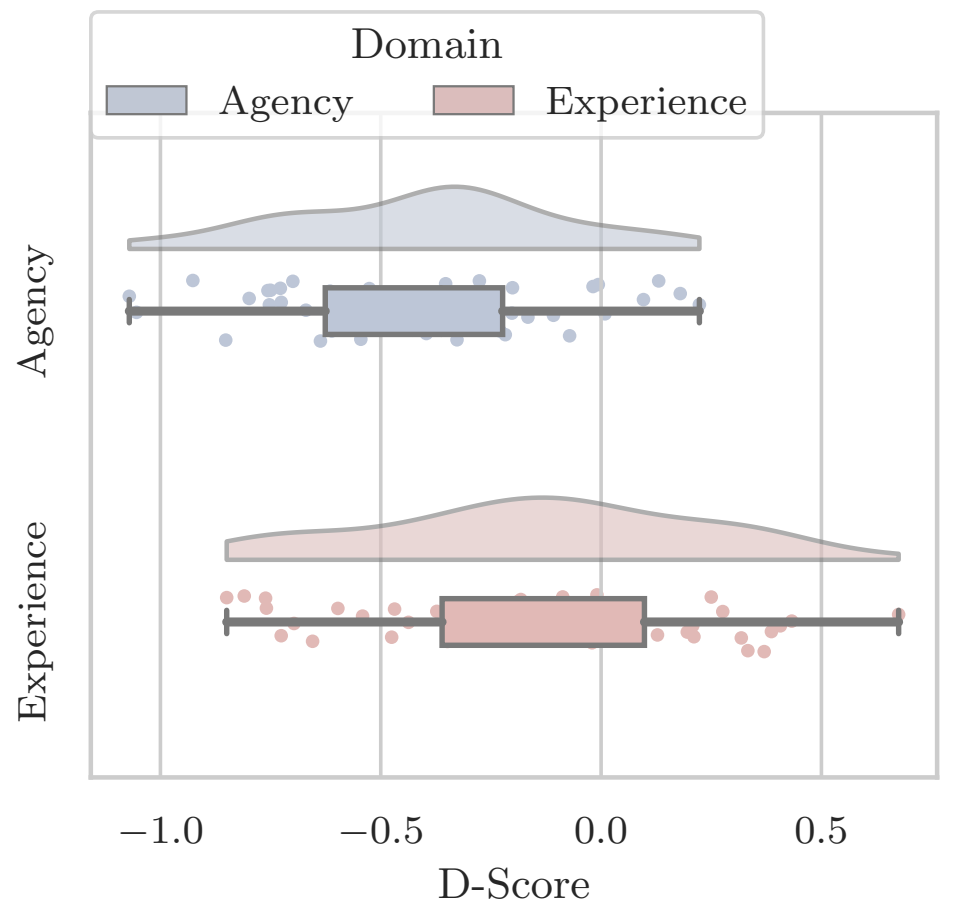

Fig. 3. Implicit measures of agency (in blue) and experience (in red). Negative D-scores indicate a higher degree of mind perception toward human agents.

\section{B. Explicit Mind Perception}

Explicit mind perception ratings were higher in both dimensions for human $\left(\mathrm{M}_{\text {agency }}=7.57, \mathrm{SD}_{\text {agency }}=0.42\right.$; $\left.\mathrm{M}_{\text {experience }}=7.95, \mathrm{SD}_{\text {experience }}=0.18\right)$ than for robotic agents $\left(\mathrm{M}_{\text {agency }}=3.01, \mathrm{SD}_{\text {agency }}=1.55 ; \mathrm{M}_{\text {experience }}=1.36\right.$, $\left.\mathrm{SD}_{\text {experience }}=0.69\right)$. Differences between agent groups were statistically significant for both agency $(\mathrm{M}=-4.55, \mathrm{SD}$ $=1.69, \mathrm{t}(50)=-19.19, p<0.001$, see Fig. 4$)$ and experience $(\mathrm{M}=-6.59, \mathrm{SD}=0.78, \mathrm{t}(50)=-59.95, p<0.001$, see Fig. 5). Paired Pitman-Morgan tests revealed significantly higher variances in both dimensions for robotic agents $\left(\mathrm{t}(49)_{\text {agency }}=(12.47), p<0.001 ; \mathrm{t}(49)_{\text {experience }}=12.45, p<0.001\right)$. Focusing on robotic agents, the variances are significantly higher for the dimension of agency $(\mathrm{t}(49)=7.28, p<0.001)$. 


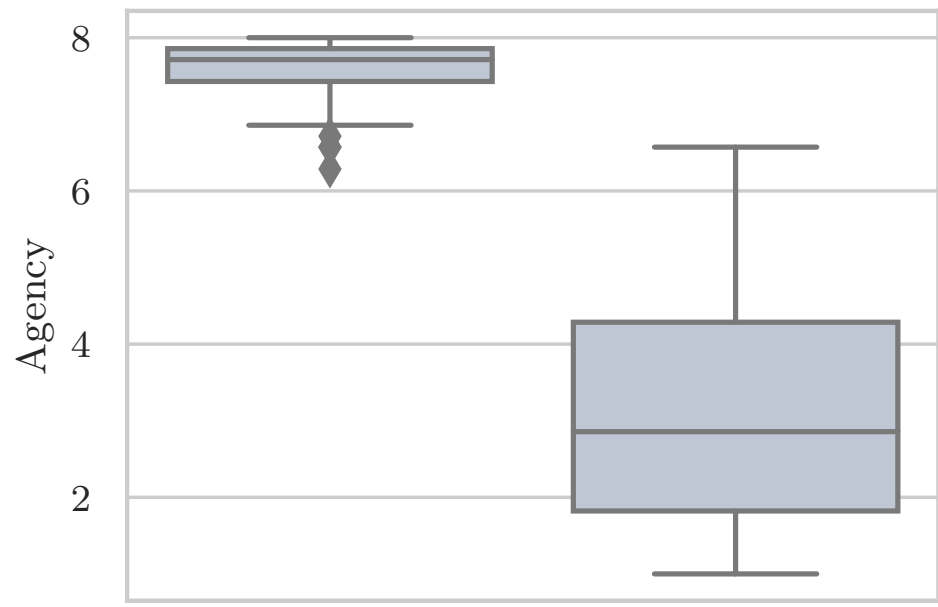

Human Robot

Agent Type

Fig. 4. Explicit ratings of agency towards human versus robot agents.

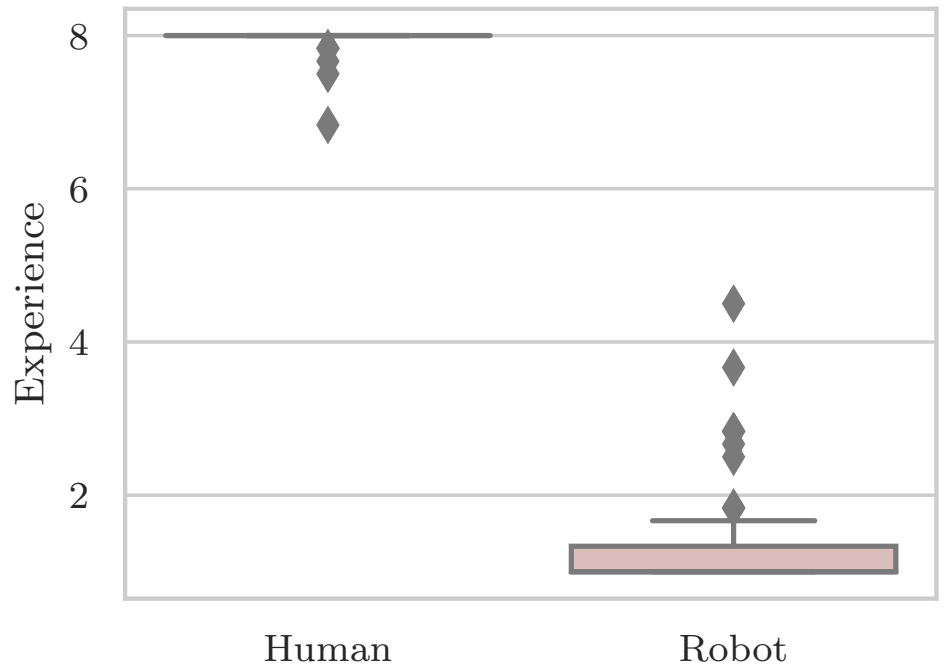

Agent Type

Fig. 5. Explicit ratings of experience towards human versus robot agents.

\section{Correlation: Implicit and Explicit Measures}

To check for the correlations between implicit and explicit mind perception scores, the D-scores were correlated with the difference of the explicit measures between human and robotic agents (explicit measure robot - explicit measure human). No relevant correlations were found ( $\mathrm{r}_{\text {agency }}=-0.09, p=0.95 ; \mathrm{r}_{\text {experience }}=0.11, p=0.44$ ). 


\section{DISCUSSION}

The goal of the current study was to examine whether (i) differences in attitudes regarding the attribution of mental capacities towards humans versus robots already exist at the subconscious level, and (ii) whether implicit and explicit measures of mind perception are correlated. For that purpose, we (i) created a customized version of the IAT (MP-IAT), suitable to assess subconscious attributions of mental capacities to human and robot agents, and (ii) used a correlation analysis to compare these implicit measures of mind perception to traditional explicit measures of mind perception using rating scales. Results indicate that (i) already at the subconscious level, robots are associated with lower levels of agency and experience than humans, and that (ii) implicit and explicit measures of mind perception are not significantly correlated. This suggests that mind perception (i) has an implicit component that can be measured using implicit tests like the MP-IAT and (ii) might be difficult to modulate via design or experimental procedures due to its fast-acting, automatic nature. The theoretical and practical implications of these findings are discussed below.

\section{A. Theoretical Contributions}

In line with previous literature [6], the explicit mind perception ratings revealed higher scores for human than for robot agents across both mind perception dimensions: human agents received almost maximal scores for both dimensions; for robots, ratings were low across the board but with higher and more variable agency than experience ratings. This suggests that participants are consistent in their explicit assumption that robot agents have a low capacity to feel and sense (i.e, experience), while beliefs regarding their capacity to act and exert self-control (i.e., agency) vary more strongly.

The implicit mind perception measures confirm this general tendency of participants to attribute less agency and experience to robot agents than to humans. Interestingly, however, when looking at implicit measures, human and robot agents seem to differ more strongly in terms of their agency rather than their experience attributions, whereas this pattern was reversed for the explicit measures (in line with previous studies using explicit measures [6], [14]). While this could indicate actual differences in implicit and explicit mind perception processes, it could also be influenced by the fact that agent stimuli in the current study were presented as full-body images instead of category labels (e.g. "human", "animal", "robot") or face images like in most studies using explicit measures of mind perception [6], [14]. The use of stimuli focusing on the physical body of an agent can modulate mind perception, such that lower levels of agency and higher levels of experience are ascribed to full-body representations compared to face representations of the same agent [55]. Although the current experiment does not allow for this hypothesis to be tested directly, it is possible that the full-body agent presentation disproportionately affected the implicit robot ratings, resulting in higher than normal experience ratings and lower than normal agency ratings for 
this stimulus category. This could easily be examined in follow-up studies repeating the current experiment either with category labels or face stimuli.

To compare explicit and implicit mind perception processes, we correlated human-robot differences in implicit mind perception measures (i.e., D-Scores for agency and experience dimensions) with corresponding humanrobot differences in explicit measures (i.e., score robotic agents - score human agents for agency and experience dimension). A general tendency of reduced levels of mind attribution to robots compared to human agents was observed. Both implicit and explicit measures show no significant correlation within participants [47]. This is most likely due to the fact that human-robot differences are more pronounced for agency than experience for the implicit measures, whereas the pattern is reversed (i.e., larger human-robot difference for experience than for agency) for the explicit measures. The results therefore indicate that implicit and explicit measures of mind perception are related but potentially independent concepts (see [48]). This is in line with accounts that describe mind perception from a dual-process perspective, such that both automatic, fast-acting cognitive processes (implicit mind perception) and slower, conscious reasoning processes (explicit mind perception) are at work when assessing the mind status of human and nonhuman agents [39].

\section{B. Practical Contributions}

The main objective of the current study was to explore whether implicit measures of mind perception can be extended to the field of social robotics, and to test whether assessments of mental capacities of human and robot agents differ on the implicit versus explicit levels. Stimuli have been carefully selected and adapted from previously published studies to create a version of the IAT that is suitable to measure implicit mind perception processes (MPIAT) based on the two-dimensional model by Gray et al.(2007) [6]. The study illustrates that implicit measures of mind perception can be indeed obtained when comparing human and robot agents. This successful demonstration of the method extends the variation of tools to measure the attribution of mind [6], [15], [16] and paves the way towards a more holistic understanding and measurement of human perception of robotic agents. The IAT provides future research with more flexibility regarding choice of stimuli (e.g., pictures vs. words) and study settings.

Mind perception has been shown to influence important aspects of social cognition relevant for HRI, such as task allocation [56], the desire to use a robot [57], or the acceptance of physical abuse and violence towards robots [58]. The current experiment indicates that mind perception potentially has separable implicit and explicit components. Although the general tendency of lower mind attribution towards robots than humans is consistent across implicit and explicit measures, differences between humans and robots seem to be accentuated on different dimensions of implicit than explicit measures. While the current experiment cannot identify the source for this dissociation, it strongly suggests that both implicit and explicit mind perception assessments should be obtained in future studies. 
The observation that mind perception has an implicit component is noteworthy in and of itself. Not only does it highlight that assessing the mental capacities of nonhuman agents has a fast-acting, automatic element that is potentially hard to explicitly modify due to its subconscious nature [39], it also suggests that it is potentially subject to biases (comparable to e.g., racial biases which are traditionally assessed via the IAT). The implicit nature of some of these beliefs might be especially influential on the behavior of inexperienced users, who have not yet formed a reflected attitude towards robots. The findings also emphasize that certain design choices alone, such as anthropomorphic design, might not be sufficient to influence peoples' beliefs about the mental capacities of robot agents, given the automatic nature of such implicit attributions.

\section{Limitations and Future Works}

Although implicit association tests have been wildly used over the last decades, there is a lot of controversy about the method's value and limitations in the scientific community. With construct validity varying strongly among different implementations, it is likely that explanatory advantage on an individual level is limited [59]. Additionally, the IAT is a measure of automatic association that not necessarily measures purely personal attitudes, but is rather also influenced by culturally acquired associations independent of their actual endorsement by the participant [60][62]. Acknowledging the critical aspect of the testing method, in line with [35], [43], [44], [63], we nevertheless consider the IAT to be a valid and valuable measure of relatively automatic cognitive classification processes at group level.

Based on the successful application of the MP-IAT in this study, some changes to the design bear the potential for improvement and further simplification of the procedure. One possible option would be an adaption of the current two-dimensional MP-IAT (based on the well established model of [6]) to a multidimensional measure inspired by [16] or [15]. This might enable a more precise division of the mental capacities of mind perception.

The selection of attribute stimuli was partially modified from previous IAT research [50], resulting in a word selection considered appropriate for the first version of an implicit measure of mind perception. Optimizing stimuli selection for the MP-IAT and mind perception questionnaire using factor analytic methods could enhance the validity of both measures for future research. A short version of the IAT (B-IAT), comprising 5 instead of 7 blocks, would lower the cognitive demands on participants without relevant influence on the results [64]. If an implicit mind perception measurement was to be combined with an additional experimental task in future studies, such adjustments would contribute to an optimal applicability. Testing the predictive power of implicit mind perception for different aspects of HRI, such as the inclination to work with or to trust a robot, appears as a natural extension of our research.

Future research could also use adapted versions of the MP-IAT to address variation in mind perception towards different robotic agents. Comparing agents with specific discrepancies in appearance or behavioural features has 
the potential to identify the influence of those features on implicit beliefs about agency and experience. The recent publication of best research practices for IAT [31] provides insightful information for any adaptation of the applied MP-IAT design. 


\section{CONCLUSION}

To our knowledge this study was the first empirical research assessing mind attribution processes towards robotic agents using an IAT. The developed MP-IAT provides a promising addition to existing methods of measuring mind perception towards agents with various degrees of human likeness. It thus widens the methodological scope of the research area and can help to paint a more complete picture of human interaction with robotic agents. Future studies could consider using different mind perception dimensions from [16] and [15], as well as evaluating social robots with other appearances and functions. The results of our study shed light on differences in mind perception between robotic and human agents. These already exist at an unconscious level and could contribute to the understanding of adaption barriers in HRI.

\section{Data AND Code Availability}

Code to implement the MP-IAT, the full dataset, tables containing the statistical results and scripts used for data analysis are publicly available at https://osf.io/v98xt/ [65]. Note that this version of the IAT does include placeholder images instead of the original stimuli.

\section{SUPPLEMENTARY MATERIAL}

A video illustrating the MP-IAT procedure (Supplementary Movie S1; also available at https://osf.io/d7q2t/) is available alongside this article.

\section{ACKNOWLEDGMENT}

We thank Patrick P. Weis for his support in providing the IAT online study framework and his tips and recommendations for programming and implementing the online experiment.

\section{REFERENCES}

[1] J. Holt-Lunstad, T. B. Smith, and J. B. Layton, "Social relationships and mortality risk: a meta-analytic review," PLoS medicine, vol. 7, no. 7, p. e1000316, 2010.

[2] B. R. Duffy, "Anthropomorphism and the social robot," Robotics and autonomous systems, vol. 42, no. 3-4, pp. 177-190, 2003.

[3] C. Breazeal, A. Takanishi, and T. Kobayashi, Social Robots that Interact with People. Berlin, Heidelberg: Springer, 2008, pp. 1349-1369. [Online]. Available: https://doi.org/10.1007/978-3-540-30301-5_59

[4] H. Brenton, M. Gillies, D. Ballin, and D. Chatting, "The uncanny valley: does it exist," in Proceedings of conference of human computer interaction, workshop on human animated character interaction. Citeseer, 2005.

[5] J. Seyama and R. S. Nagayama, "The uncanny valley: Effect of realism on the impression of artificial human faces," Presence, vol. 16, no. 4, pp. 337-351, 2007. 
[6] H. M. Gray, K. Gray, and D. M. Wegner, "Dimensions of Mind Perception,” Science, vol. 315, no. 5812, pp. 619-619, Feb. 2007. [Online]. Available: https://www.sciencemag.org/lookup/doi/10.1126/science.1134475

[7] S. Almaraz, K. Hugenberg, and S. Young, "Perceiving sophisticated minds influences perceptual individuation," Personality and Social Psychology Bulletin, vol. 44, pp. 143-157, 2017.

[8] J. Graham and J. Haidt, "Beyond beliefs: Religions bind individuals into moral communities," Personality and Social Psychology Review, vol. 14, no. 1, pp. 140-150, 2010, pMID: 20089848. [Online]. Available: https://doi.org/10.1177/1088868309353415

[9] A. Druin and J. Hendler, Eds., Robots for Kids: Exploring New Technologies for Learning. San Francisco, CA, USA: Morgan Kaufmann Publishers Inc., 2000.

[10] E. Rosset, "It's no accident: Our bias for intentional explanations," Cognition, vol. 108, pp. 771-80, Nov. 2008.

[11] A. Waytz, J. Cacioppo, and N. Epley, "Who Sees Human? The Stability and Importance of Individual Differences in Anthropomorphism," Perspectives on psychological science : a journal of the Association for Psychological Science, vol. 5, no. 3, pp. 219-232, 2010.

[12] K. Urbanska, "Review of the book'the mind club: Who thinks, what feels and why it matters' by dm wegner \& k. gray," Social Psychological Review, vol. 18, pp. 57-59, 2016.

[13] N. Haslam, "Dehumanization: An integrative review," Personality and social psychology review, vol. 10, no. 3, pp. 252-264, 2006.

[14] M. Tharp, N. S. Holtzman, and F. R. Eadeh, "Mind Perception and Individual Differences: A Replication and Extension," Basic and Applied Social Psychology, vol. 39, no. 1, pp. 68-73, Jan. 2017. [Online]. Available: https://www.tandfonline.com/doi/full/10.1080/ 01973533.2016 .1256287

[15] K. Weisman, C. S. Dweck, and E. M. Markman, "Rethinking people's conceptions of mental life," Proceedings of the National Academy of Sciences, vol. 114, no. 43, pp. 11374-11379, 2017.

[16] B. Malle, "How many dimensions of mind perception really are there?" in Proceedings of the 41st Annual Conference of the Cognitive Science Society. Montreal, QB: Cognitive Science Society, 2019, pp. 2268-2274.

[17] K. Haley and D. Fessler, “Nobody's watching? subtle cues affect generosity in,” Evolution and Human Behavior, vol. 26, p. 245-256, 2005 .

[18] N. Epley, A. Waytz, S. Akalis, and J. Cacioppo, "When we need a human: Motivational determinants of anthropomorphism," Social Cognition - SOC COGNITION, vol. 26, pp. 143-155, 2008.

[19] N. Hertz and E. Wiese, "Under pressure: Examining social conformity with computer and robot groups," Human factors, vol. 60, no. 8, pp. 1207-1218, 2018.

[20] A. Abubshait, A. Momen, and E. Wiese, "Seeing human: Do individual differences modulate the uncanny valley?" Proceedings of the Human Factors and Ergonomics Society Annual Meeting, vol. 61, no. 1, pp. 870-874, 2017.

[21] P. P. Weis and E. Wiese, "Cognitive conflict as possible origin of the uncanny valley," Proceedings of the Human Factors and Ergonomics Society Annual Meeting, vol. 61, no. 1, pp. 1599-1603, 2017. [Online]. Available: https://doi.org/10.1177/1541931213601763

[22] C. E. Looser and T. Wheatley, "The Tipping Point of Animacy: How, When, and Where We Perceive Life in a Face," Psychological Science, vol. 21, no. 12, pp. 1854-1862, Dec. 2010. [Online]. Available: http://journals.sagepub.com/doi/10.1177/0956797610388044

[23] J. Kätsyri, K. Förger, M. Mäkäräinen, and T. Takala, “A review of empirical evidence on different uncanny valley hypotheses: support for perceptual mismatch as one road to the valley of eeriness," Frontiers in Psychology, vol. 6, p. 390, 2015. [Online]. Available: https://www.frontiersin.org/article/10.3389/fpsyg.2015.00390

[24] M. Cheetham, P. Suter, and L. Jancke, "Perceptual discrimination difficulty and familiarity in the uncanny valley: more like a "happy valley"," Frontiers in Psychology, vol. 5, p. 1219, 2014. [Online]. Available: https://www.frontiersin.org/article/10.3389/fpsyg.2014. 01219

[25] A. P. Saygin, T. Chaminade, H. Ishiguro, J. Driver, and C. Frith, "The thing that should not be: predictive coding and the uncanny valley in perceiving human and humanoid robot actions," Social cognitive and affective neuroscience, vol. 7, pp. 413-22, Apr. 2011. 
[26] E. Wiese, A. Mandell, T. Shaw, and M. Smith, "Implicit mind perception alters vigilance performance because of cognitive conflict processing." Journal of experimental psychology: applied, vol. 25, no. 1, p. 25, 2019.

[27] F. Castelli, F. Happe, U. Frith, and C. Frith, "Movement and mind: A functional imaging study of perception and interpretation of complex intentional movement patterns," NeuroImage, vol. 12, no. 3, pp. 314-325, 2000.

[28] S. Tulk, R. Cumings, T. Zafar, and E. Wiese, "Better know who you are starving with: Judging humanness in a multiplayer videogame," in Proceedings of the Technology, Mind, and Society, ser. TechMindSociety '18. New York, NY, USA: Association for Computing Machinery, 2018. [Online]. Available: https://doi.org/10.1145/3183654.3183710

[29] A. Wykowska, R. Chellali, M. M. Al-Amin, and H. J. Müller, "Implications of robot actions for human perception. how do we represent actions of the observed robots?" International Journal of Social Robotics, vol. 6, no. 3, pp. 357-366, 2014.

[30] A. Wykowska, T. Chaminade, and G. Cheng, "Embodied artificial agents for understanding human social cognition," Philosophical Transactions of the Royal Society B: Biological Sciences, vol. 371, no. 1693, p. 20150375, 2016. [Online]. Available: https://royalsocietypublishing.org/doi/abs/10.1098/rstb.2015.0375

[31] A. G. Greenwald, M. Brendl, H. Cai, D. Cvencek, J. F. Dovidio, M. Friese, A. Hahn, E. Hehman, W. Hofmann, S. Hughes et al., "Best research practices for using the implicit association test," Behavior research methods, pp. 1-20, 2021.

[32] C. M. Carpinella, A. B. Wyman, M. A. Perez, and S. J. Stroessner, "The Robotic Social Attributes Scale (RoSAS): Development and Validation," in Proceedings of the 2017 ACM/IEEE International Conference on Human-Robot Interaction. Vienna Austria: ACM, Mar. 2017, pp. 254-262. [Online]. Available: https://dl.acm.org/doi/10.1145/2909824.3020208

[33] T. Nomura, T. Suzuki, T. Kanda, and K. Kato, "Measurement of negative attitudes toward robots," Interaction Studies. Social Behaviour and Communication in Biological and Artificial Systems, vol. 7, no. 3, pp. 437-454, Nov. 2006. [Online]. Available: http://www.jbe-platform.com/content/journals/10.1075/is.7.3.14nom

[34] K. F. MacDorman, S. K. Vasudevan, and C.-C. Ho, "Does Japan really have robot mania? Comparing attitudes by implicit and explicit measures," AI \& SOCIETY, vol. 23, no. 4, pp. 485-510, Jul. 2009. [Online]. Available: http://link.springer.com/10.1007/s00146-008-0181-2

[35] A. G. Greenwald, D. E. McGhee, and J. L. K. Schwartz, "Measuring individual differences in implicit cognition: The implicit association test." Journal of Personality and Social Psychology, vol. 74, no. 6, pp. 1464-1480, 1998. [Online]. Available: http://doi.apa.org/getdoi.cfm?doi=10.1037/0022-3514.74.6.1464

[36] S. Loughnan and N. Haslam, "Animals and androids: implicit associations between social categories and nonhumans," Psychological science, vol. 18, no. 2, pp. 116-121, 2007

[37] B. Gawronski and G. V. Bodenhausen, "Associative and propositional processes in evaluation: An integrative review of implicit and explicit attitude change." Psychological Bulletin, vol. 132, no. 5, pp. 692-731, Sep. 2006. [Online]. Available: http://doi.apa.org/getdoi.cfm?doi=10.1037/0033-2909.132.5.692

[38] E. G. Urquiza-Haas and K. Kotrschal, "The mind behind anthropomorphic thinking: attribution of mental states to other species," Animal Behaviour, vol. 109, pp. 167-176, Nov. 2015. [Online]. Available: https://doi.org/10.1016/j.anbehav.2015.08.011

[39] T. D. Wilson, S. Lindsey, and T. Y. Schooler, “A model of dual attitudes.” Psychological Review, vol. 107, no. 1, pp. 101-126, 2000. [Online]. Available: http://doi.apa.org/getdoi.cfm?doi=10.1037/0033-295X.107.1.101

[40] S. M. Merritt, H. Heimbaugh, J. LaChapell, and D. Lee, "I trust it, but I don't know why: effects of implicit attitudes toward automation on trust in an automated system," Human factors, vol. 55, no. 3, pp. 520-534, 2013.

[41] S.-E. Chien, L. Chu, H.-H. Lee, C.-C. Yang, F.-H. Lin, P.-L. Yang, T.-M. Wang, and S.-L. Yeh, “Age Difference in Perceived Ease of Use, Curiosity, and Implicit Negative Attitude toward Robots," ACM Transactions on Human-Robot Interaction, vol. 8, no. 2, pp. 1-19, 2019. [Online]. Available: https://dl.acm.org/doi/10.1145/3311788

[42] M. M. de Graaf, S. Ben Allouch, and S. Lutfi, "What are people's associations of domestic robots?: Comparing implicit and explicit 
measures," in 2016 25th IEEE International Symposium on Robot and Human Interactive Communication (RO-MAN). New York, NY, USA: IEEE, Aug. 2016, pp. 1077-1083. [Online]. Available: http://ieeexplore.ieee.org/document/7745242/

[43] T. L. Sanders, K. E. Schafer, W. Volante, A. Reardon, and P. A. Hancock, "Implicit Attitudes Toward Robots," Proceedings of the Human Factors and Ergonomics Society Annual Meeting, vol. 60, no. 1, pp. 1746-1749, 2016.

[44] N. Spatola and O. A. Wudarczyk, "Implicit attitudes towards robots predict explicit attitudes, semantic distance between robots and humans, anthropomorphism, and prosocial behavior: From attitudes to human-robot interaction," International Journal of Social Robotics, vol. 13, no. 5, pp. 1149-1159, 2021.

[45] — _ "Ascribing emotions to robots: Explicit and implicit attribution of emotions and perceived robot anthropomorphism," Computers in Human Behavior, vol. 124, p. 106934, 2021.

[46] J. Złotowski, H. Sumioka, F. Eyssel, S. Nishio, C. Bartneck, and H. Ishiguro, "Model of Dual Anthropomorphism: The Relationship Between the Media Equation Effect and Implicit Anthropomorphism,” International Journal of Social Robotics, vol. 10, no. 5, pp. 701-714, Nov. 2018. [Online]. Available: http://link.springer.com/10.1007/s12369-018-0476-5

[47] J. Banks, "Theory of mind in social robots: replication of five established human tests," International Journal of Social Robotics, vol. 12 , no. 2 , pp. 403-414, 2020 .

[48] B. A. Nosek, "Implicit-Explicit Relations," Current Directions in Psychological Science, vol. 16, no. 2, pp. 65-69, Apr. 2007. [Online]. Available: http://journals.sagepub.com/doi/10.1111/j.1467-8721.2007.00477.x

[49] H. Blanton, J. Jaccard, and C. N. Burrows, "Implications of the implicit association test d-transformation for psychological assessment," Assessment, vol. 22, no. 4, pp. 429-440, 2015.

[50] W. K. Campbell, J. K. Bosson, T. W. Goheen, C. E. Lakey, and M. H. Kernis, "Do narcissists dislike themselves deep down inside?" Psychological science, vol. 18, no. 3, pp. 227-229, 2007.

[51] A. Smeding, "Women in Science, Technology, Engineering, and Mathematics (STEM): An Investigation of Their Implicit Gender Stereotypes and Stereotypes' Connectedness to Math Performance," Sex Roles, vol. 67, no. 11-12, pp. 617-629, 2012.

[52] E. Phillips, X. Zhao, D. Ullman, and B. F. Malle, "What is Human-like?: Decomposing Robots' Human-like Appearance Using the Anthropomorphic roBOT (ABOT) Database," in Proceedings of the 2018 ACM/IEEE International Conference on Human-Robot Interaction. Chicago IL USA: ACM, Feb. 2018, pp. 105-113. [Online]. Available: https://dl.acm.org/doi/10.1145/3171221.3171268

[53] A. G. Greenwald, B. A. Nosek, and M. R. Banaji, "Understanding and using the Implicit Association Test: I. An improved scoring algorithm." Journal of Personality and Social Psychology, vol. 85, no. 2, pp. 197-216, 2003. [Online]. Available: http://doi.apa.org/getdoi.cfm?doi=10.1037/0022-3514.85.2.197

[54] M. Allen, D. Poggiali, K. Whitaker, T. R. Marshall, J. van Langen, and R. A. Kievit, "Raincloud plots: a multiplatform tool for robust data visualization," Wellcome Open Research, vol. 4, p. 63, Jan. 2021. [Online]. Available: https://wellcomeopenresearch.org/articles/4-63/v2

[55] K. Gray, J. Knobe, M. Sheskin, P. Bloom, and L. F. Barrett, "More than a body: mind perception and the nature of objectification." Journal of personality and social psychology, vol. 101, no. 6, p. 1207, 2011.

[56] E. Wiese, P. P. Weis, Y. Bigman, K. Kapsaskis, and K. Gray, "It's a match: Task assignment in human-robot collaboration depends on mind perception," International Journal of Social Robotics, Mar. 2021. [Online]. Available: https://doi.org/10.1007/s12369-021-00771-z

[57] R. Q. Stafford, B. A. MacDonald, C. Jayawardena, D. M. Wegner, and E. Broadbent, "Does the robot have a mind? mind perception and attitudes towards robots predict use of an eldercare robot," International Journal of Social Robotics, vol. 6, no. 1, pp. 17-32, Apr. 2013. [Online]. Available: https://doi.org/10.1007/s12369-013-0186-y

[58] M. Keijsers, C. Bartneck, and F. Eyssel, "Pay them no mind: the influence of implicit and explicit robot mind perception on the right to be protected," International Journal of Social Robotics, Jul. 2021. [Online]. Available: https://doi.org/10.1007/s12369-021-00799-1 
[59] U. Schimmack, "The Implicit Association Test: A Method in Search of a Construct," Perspectives on Psychological Science, vol. 16, no. 2, pp. 396-414, Mar. 2021. [Online]. Available: http://journals.sagepub.com/doi/10.1177/1745691619863798

[60] A. Karpinski and J. L. Hilton, "Attitudes and the Implicit Association Test.” Journal of Personality and Social Psychology, vol. 81, no. 5, pp. 774-788, Nov. 2001. [Online]. Available: http://doi.apa.org/getdoi.cfm?doi=10.1037/0022-3514.81.5.774

[61] R. Fazio and M. Olson, "Implicit Measures in Social Cognition Research: Their Meaning and Use," Annual review of psychology, vol. 54, pp. 297-327, Feb. 2003.

[62] H. R. Arkes and P. Tetlock, "Attributions of implicit prejudice, or "would jesse jackson 'fail' the implicit association test?"," Psychological Inquiry, vol. 15, pp. 257 - 278, 2004.

[63] B. Kurdi, K. A. Ratliff, and W. A. Cunningham, "Can the Implicit Association Test Serve as a Valid Measure of Automatic Cognition? A Response to Schimmack (2021),” Perspectives on Psychological Science, vol. 16, no. 2, pp. 422-434, Mar. 2021. [Online]. Available: http://journals.sagepub.com/doi/10.1177/1745691620904080

[64] N. Sriram and A. G. Greenwald, "The Brief Implicit Association Test,” Experimental Psychology, vol. 56, no. 4, pp. 283-294, Jan. 2009. [Online]. Available: https://econtent.hogrefe.com/doi/10.1027/1618-3169.56.4.283

[65] J. P. Woller, L. Terfurth, Z. Li, and E. Wiese, "Mind perception implicit association test (mp-iat)," Jan 2022. [Online]. Available: osf.io/v98xt 